\title{
THE RELATION OF HIGH AND LOW UREA CLEARANCES TO THE INULIN AND CREATININE CLEARANCES IN CHIL- DREN WITH THE NEPHROTIC SYNDROME
}

\author{
By KENDALL EMERSON, JR., PALMER H. FUTCHER, AND LEE E. FARR \\ (From the Hospital of The Rockefeller Institute for Medical Research, New York City)
}

(Received for publication March 13, 1941)

During the past 6 years we have observed that, in children with the nephrotic syndrome, the urea clearance is not infrequently increased for periods of 1 or more months to 140 per cent or more of the average clearance of normal children of similar age and size. The phenomenon has occurred with the same frequency in both sexes. In a group of 33 nephrotic children all less than 10 years of age who were admitted to the Hospital, we have seen this type of elevation in 14 (or 42 per cent) of the patients. In 6 of the 14 patients the elevation has persisted for a period of at least 6 months; in 1 of our nephrotic children the urea clearance has been consistently elevated to between 200 and 300 per cent of normal for 6 years. These nephrotic children have been found by Farr (1) to show also a degree of lability of the urea clearance not noted in normal adult man (2) or, in our experience and in that of others, in children or adults (3) with decreased urea clearances. When the dietary protein was reduced from the optimum intake of 3 grams per kilo to 1 gram or less, the urea clearance showed a parallel fall. In contrast to our young children, only 2 out of a group of 54 nephrotic adults and children over 10 years of age observed in this Hospital have shown high clearances; these were aged 11 and 18 years.

The mechanism of this high urea clearance in nephrotic children has not been explained. In the present study we have sought to determine whether the increased urea clearance is accompanied by a similar increase in the inulin clearance, which is believed to equal the volume of the glomerular filtrate (4). We have also determined the ratios of inulin clearance to urea clearance and to creatinine clearance in these patients, and compared them with the same ratios in nephrotic children with diminished urea clearances, and in children who have recovered from acute nephritis
Patients studied and experimental procedures followed

The patients studied in our experiments were 3 nephrotic children (R. Q., S. G., R. M.) with high urea clearances, 2 nephrotic children (J. C., S. W.) and 1 nephrotic adult (A. C.) with low urea clearances and, as controls with normal renal function, 2 children who had recovered from acute hemorrhagic Bright's disease. Of the 3 patients in the high clearance group, 1, R. M., had a urea clearance consistently elevated to above 140 per cent of normal; the other 2 patients had urea clearances always above 100 per cent of normal and frequently above 140 per cent. All the nephrotic patients exhibited proteinuria and hyperlipemia and had plasma albumin levels below 2.5 grams per $100 \mathrm{cc}$. Edema had been present previously in each case but was observed only in S. G. at the time of these experiments. Detailed laboratory and clinical data on 4 of these patients (R. Q., S. G., J. C., S. W.) have been published elsewhere $(5,6)$. For several months previous to these experiments all of the patients except B. D. and A. C. had been fed a diet which provided 3 grams of protein per kilogram of ideal body weight. The daily intake of sodium chloride was 1 to 1.5 grams.

All tests were performed under fasting conditions; the subjects were kept in bed during the clearance periods. Preceding and during the experiments, from 1 to 2 liters of water were administered orally to maintain an adequate flow of urine. The patients were not catheterized. After 2 or 3 control periods, each of approximately 1 hour's duration, during which specimens of urine and a single blood sample were obtained for the determination of urea and "endogenous" creatinine clearances, creatinine was administered orally. One hour later a single injection of inulin, prepared as a 10 per cent solution in 0.85 per cent sodium chloride solution, 1 was administered intravenously during the course of 15 to 20 minutes. The quantities of creatinine and inulin given varied in the individual experiments as shown in Tables I, II and III. Thirty to 60 minutes following the injection of inulin, urine collections were resumed for the determination of simultaneous inulin, "exogenous" creatinine, and urea clearances. The duration of these fatter periods of urine collection varied usually from 30 to 60 minutes and was determined by the patients' desire to void. Venous blood samples were obtained at the beginning and end of each period.

1 The 10 per cent solution of inulin in saline was purchased from the U. S. Standard Products Co., Woodworth, Wisconsin. 
TABLE I

Nephrotic patients with high urea clearances. Comparison of inulin, creatinine, and urea clearances

\begin{tabular}{|c|c|c|c|c|c|c|c|c|c|c|c|c|c|c|c|}
\hline \multirow[b]{2}{*}{ Subject } & \multirow[b]{2}{*}{ Period } & \multirow[b]{2}{*}{$\underset{\text { tion }}{\text { Dura- }}$} & \multirow[b]{2}{*}{$\begin{array}{c}\text { Urine } \\
\text { flow } \\
V \ddagger \ddagger\end{array}$} & \multicolumn{3}{|c|}{ Plasma levels } & \multicolumn{3}{|c|}{ Urine levels } & \multicolumn{4}{|c|}{ Clearances } & \multicolumn{2}{|c|}{$\begin{array}{l}\text { Clearance } \\
\text { ratios }\end{array}$} \\
\hline & & & & Inulin & $\begin{array}{l}\text { Creat- } \\
\text { inine }\end{array}$ & $\stackrel{\text { Urea }}{\mathbf{N}}$ & Inulin & $\begin{array}{l}\text { Creat- } \\
\text { inine }\end{array}$ & $\begin{array}{c}\text { Urea } \\
\mathbf{N}\end{array}$ & Inulin & $\begin{array}{l}\text { Endog- } \\
\text { enous } \\
\text { creat- } \\
\text { inine }\end{array}$ & $\begin{array}{l}\text { Exog- } \\
\text { enous } \\
\text { creat- } \\
\text { inine }\end{array}$ & Urea & $\begin{array}{l}\text { Exog- } \\
\text { enous } \\
\text { creat- } \\
\text { inine: } \\
\text { Inulin }\end{array}$ & $\begin{array}{l}\text { Urea: } \\
\text { Inulin }\end{array}$ \\
\hline \multirow[t]{2}{*}{$\begin{array}{l}\text { R. Q. } ~ \\
8 \text { years } \\
\text { ( } V \text { factor } \neq \ddagger=1.74) \\
\text { January } 25,1940\end{array}$} & $\begin{array}{l}1 \\
2 \S \\
3 \\
4\end{array}$ & \begin{tabular}{|c|} 
minutes \\
\\
60 \\
55 \\
60 \\
60
\end{tabular} & $\begin{array}{c}c c . \\
\text { per } \\
\text { minute } \\
3.61 \\
4.30 \\
6.68 \\
3.55\end{array}$ & $\begin{array}{c}\text { mgm. } \\
\text { per } \\
100 \\
c c . \\
\\
\\
11.6 \\
4.4\end{array}$ & $\begin{array}{c}\text { mgm. } \\
\text { per } \\
100 \\
c c .\end{array}$ & $\begin{array}{c}\text { mgm. } \\
\text { per } \\
100 \\
c c . \\
7.5^{*} \\
6.2^{*} \\
6.2^{*} \\
6.4^{*}\end{array}$ & $\begin{array}{c}\text { mgm. } \\
\text { per } \\
100 \\
c c . \\
\\
\\
330 \\
280\end{array}$ & $\begin{array}{l}\text { mgm. } \\
\text { per } \\
100 \\
c c .\end{array}$ & $\begin{array}{c}\text { mgm. } \\
\text { per } \\
100 \\
c c . \\
230^{*} \\
177^{*} \\
105^{*} \\
192^{*}\end{array}$ & $\begin{array}{c}c c . \\
\text { plasma } \\
\text { per } \\
\text { minute }\end{array} \mid$ & $\begin{array}{c}c c . \\
\text { plasma } \\
\text { per } \\
\text { minute }\end{array}$ & $\begin{array}{c}c c . \\
\text { plasma } \\
\text { per } \\
\text { minute }\end{array}$ & $\begin{array}{c}c c . \\
\text { plasma } \\
\text { per } \\
\text { minute } \\
111^{*} \\
122^{*} \\
113^{*} \\
106^{*}\end{array}$ & & $\begin{array}{l}0.59^{*} \\
0.47^{*}\end{array}$ \\
\hline & \multicolumn{3}{|c|}{ Average } & & 208 & & & 113 & & \\
\hline \multirow[t]{2}{*}{$\begin{array}{l}\text { Idem } \\
\text { March 11, } 1940\end{array}$} & $\begin{array}{l}1 \\
2 \\
3 \\
49 \\
5 \| \\
6 \\
7 \\
8 \\
9\end{array}$ & $\begin{array}{l}70 \\
57 \\
58 \\
59 \\
75 \\
33 \\
32 \\
41 \\
34\end{array}$ & $\begin{array}{l}6.72 \\
3.56 \\
4.14 \\
2.32 \\
6.10 \\
1.03 \\
2.40 \\
3.84 \\
7.64\end{array}$ & $\begin{array}{r}21.0 \\
14.6 \\
10.4 \\
7.2\end{array}$ & $\begin{array}{l}0.33 \\
\\
3.45 \\
7.38 \\
5.45 \\
4.55 \\
3.78 \\
3.00\end{array}$ & $\begin{array}{l}7.6 \\
\\
7.5 \\
7.0 \\
7.0 \\
6.5 \\
6.5 \\
7.2\end{array}$ & $\begin{array}{r}4300 \\
1200 \\
450 \\
185\end{array}$ & $\begin{array}{r}9.2 \\
15.6 \\
14.0 \\
117.4 \\
340.0 \\
2030.0 \\
586.0 \\
220.0 \\
114.0\end{array}$ & $\begin{array}{l}146 \\
194 \\
208 \\
282 \\
105 \\
418 \\
256 \\
128 \\
103\end{array}$ & $\begin{array}{l}212 \\
197 \\
166 \\
196\end{array}$ & $\begin{array}{l}184 \\
166 \\
173\end{array}$ & $\begin{array}{l}281 \\
386 \\
309 \\
223 \\
290\end{array}$ & $\begin{array}{r}129 \\
91 \\
113 \\
87 \\
92 \\
86 \\
95 \\
75 \\
110\end{array}$ & $\begin{array}{l}1.82 \\
1.57 \\
1.34 \\
1.48\end{array}$ & $\begin{array}{l}0.41 \\
0.48 \\
0.45 \\
0.56\end{array}$ \\
\hline & \multicolumn{3}{|c|}{ Average } & & & & & & & 193 & 174 & 297 & 98 & & \\
\hline \multirow[t]{2}{*}{$\begin{array}{l}\text { S. G. } \sigma^{\top} \\
10 \text { years } \\
(V \text { factor } \ddagger \ddagger=1.75 \text { ) } \\
\text { February } 26,1940\end{array}$} & $\begin{array}{l}1 \\
2 \\
39 \\
4 \| \\
5 \\
6 \\
7 \dagger \dagger \\
8\end{array}$ & $\begin{array}{r}114 \\
61 \\
60 \\
60 \\
60 \\
60 \\
62 \\
58\end{array}$ & $\begin{array}{l}2.01 \\
4.70 \\
3.04 \\
6.25 \\
1.81 \\
1.20 \\
0.62 \\
1.69\end{array}$ & $\begin{array}{r}18.1 \\
8.7 \\
5.6 \\
3.2\end{array}$ & $\begin{array}{l}0.28 \\
2.30 \\
3.67 \\
2.26 \\
1.32 \\
0.95 \\
0.75\end{array}$ & $\begin{array}{r}9.1 \\
\\
8.9 \\
9.1 \\
9.4 \\
12.8 \\
14.4\end{array}$ & $\begin{array}{r}1520 \\
1380 \\
1160 \\
620\end{array}$ & $\begin{array}{r}24.7 \\
11.7 \\
295.0 \\
216.0 \\
420.0 \\
440.0 \\
403.0 \\
123.0\end{array}$ & $\begin{array}{l}418 \\
202 \\
262 \\
123 \\
346 \\
468 \\
316 \\
478\end{array}$ & $\begin{array}{l}152 \\
190 \\
129+\dagger \\
327\end{array}$ & $\begin{array}{l}177 \\
197\end{array}$ & $\begin{array}{l}390 \ddagger \\
368 \\
336 \\
370 \\
263 \dagger t \\
276\end{array}$ & $\begin{array}{c}92 \\
104 \\
88 \\
86 \\
72 \\
77 \\
28+\dagger \\
61\end{array}$ & $\begin{array}{l}2.21 \\
1.95 \\
2.04 \\
0.84\end{array}$ & $\begin{array}{l}0.47 \\
0.41 \\
0.22 \\
0.19\end{array}$ \\
\hline & \multicolumn{3}{|c|}{ Average } & & & & & & & 171 & 187 & 366 & 86 & & \\
\hline \multirow[t]{2}{*}{$\begin{array}{l}\text { R. M. } \sigma^{\top} \\
3 \text { years } \\
(V \text { factor } \ddagger \neq=2.75) \\
\text { April } 16,1940\end{array}$} & $\begin{array}{c}1 \\
2 \\
3 \\
4^{* *} \\
5 \| \\
6 \\
7 \\
8 \\
9 \\
10\end{array}$ & $\begin{array}{l}55 \\
60 \\
58 \\
52 \\
50 \\
35 \\
30 \\
30 \\
45 \\
30\end{array}$ & $\begin{array}{r}2.99 \\
11.10 \\
6.55 \\
3.59 \\
7.05 \\
10.80 \\
6.96 \\
1.46 \\
7.53 \\
5.68\end{array}$ & $\begin{array}{r}51.5 \\
20.0 \\
14.3 \\
9.8 \\
5.0\end{array}$ & $\begin{array}{c}t \\
\\
3.60 \\
11.96 \\
11.12 \\
9.40 \\
7.05 \\
5.26 \\
3.70\end{array}$ & $\begin{array}{l}6.7 \\
6.7 \\
6.6 \\
6.6 \\
6.7 \\
7.0\end{array}$ & $\begin{array}{r}1030 \\
760 \\
2300 \\
270 \\
200\end{array}$ & \begin{tabular}{|r|}
334.0 \\
634.0 \\
358.0 \\
400.0 \\
1672.0 \\
195.0 \\
170.0
\end{tabular} & $\begin{array}{r}323 \\
76 \\
116 \\
207 \\
101 \\
72 \\
95 \\
467 \\
104 \\
104\end{array}$ & $\begin{array}{l}216 \\
265 \\
236 \\
207 \\
237\end{array}$ & $\dagger$ & $\begin{array}{l}333 \ddagger \\
373 \\
349 \\
296 \\
348 \\
279 \\
261\end{array}$ & $\begin{array}{r}136 \\
119 \\
103 \\
105 \\
105 \\
115 \\
100 \\
121 \\
116 \\
85\end{array}$ & $\begin{array}{l}1.62 \\
1.12 \\
1.47 \\
1.35 \\
1.10\end{array}$ & $\begin{array}{l}0.53 \\
0.38 \\
0.51 \\
0.56 \\
0.36\end{array}$ \\
\hline & \multicolumn{3}{|c|}{ Average } & & & & & & & 233 & & 320 & 111 & & \\
\hline
\end{tabular}

* In these experiments, whole blood and urine were analyzed for urea-plus-ammonia nitrogen by the gasometric hypobromite method. The use of the hypobromite method in analyzing whole blood and urine has been demonstrated in our high-clearance patients to furnish whole blood clearance results not deviating by more than 10 per cent from simultaneous plasma clearance determinations in which plasma and urine were analyzed by the urease method. Hence, all clearance values have been tabulated as "plasma" clearances, and used as such in calculating ratios.

$\dagger$ Endogenous plasma creatinine too low to measure.

$\ddagger$ Plasma creatinine level rising during this period.

Inulin 5 grams given intravenously during this period.

II Inulin 10 grams given intravenously during this period.

I Creatinine 4 grams given by mouth during this period.

** Creatinine 5 grams given by mouth during this period.

t† Casein hydrolysate 20 grams given intravenously during this period, with subsequent chill and rise of temperature to $103.4^{\circ}$. Clearance values in Periods 7 and 8 not used in calculating averages.

$\ddagger$ To obtain " $V$," which is the urine flow per minute per 1.73 square meters of body surface, the observed urine flow has been multiplied by the " $V$ factor," which is the ratio of 1.73 to the subject's surface area in square meters, determined from his age and height (15). 
Casein hydrolysate, ${ }^{2}$ prepared as a 10 per cent solution $(6,7)$ was given intravenously on at least one occasion to each of the low-clearance patients, and to S. G. in the high-clearance group. The amino acid mixture was given after 1 or 2 periods of simultaneous determination of inulin, urea, and creatinine clearances, and all clearances were again determined during 1 or 2 subsequent periods. The quantities of casein hydrolysate given to each patient are shown in Tables I and II.

\section{ANALYTICAL METHODS}

Urea-plus-ammonia nitrogen in whole blood and urine was determined in some experiments by the hypobromite gasometric method of Van Slyke and Kugel (8); the experiments in which this method was used are indicated by an asterisk in Tables I and II. In the remainder plasma and urine urea nitrogen were determined by the gasometric urease method of Van Slyke (9).

${ }^{2}$ Furnished through the generosity of Mead Johnson and Co., Evansville, Indiana.
Creatinine was determined with the Summerson (10) photoelectric colorimeter by the method of Folin and Wu as modified by Miller and Winkler (11). The plasma values of the 2 control patients were corrected for noncreatinine chromogen by the specific enzymatic method of Miller and Dubos (12). In the high-clearance group the "endogenous" plasma chromogen levels were so low as to make accurate determination of non-creatinine chromogen impossible; indeed, in the case of R. M. there was no Jaffe reaction demonstrable in the plasma filtrate. In the single low-clearance patient (J. C.) in which it was determined, the non-creatinine chromogen was less than 10 per cent of the total chromogenic substance of the plasma; no corrections have been applied to the plasma values of the low-clearance group.

Plasma and urinary inulin were determined by the technique described by Alving, Rubin and Miller (13), which was modified slightly according to suggestions made by $\mathrm{Dr}$. A. S. Alving in personal communications; the Summerson photoelectric colorimeter was employed.

TABLE II

Nephrotic patients with low urea clearances. Comparison of inulin, creatinine, and urea clearances

\begin{tabular}{|c|c|c|c|c|c|c|c|c|c|c|c|c|c|c|c|c|}
\hline \multirow[b]{2}{*}{ Subject } & \multirow[b]{2}{*}{ Period } & \multirow[b]{2}{*}{$\begin{array}{l}\text { Dur- } \\
\text { ation }\end{array}$} & \multirow[b]{2}{*}{$\begin{array}{l}\text { Urine } \\
\text { flow } \\
V \& \$\end{array}$} & \multicolumn{3}{|c|}{ Plasma levels } & \multicolumn{3}{|c|}{ Urine levels } & \multicolumn{4}{|c|}{ Clearances } & \multicolumn{3}{|c|}{ Clearance ratios } \\
\hline & & & & $\ln _{\ln }$ & $\begin{array}{c}\text { Cre- } \\
\text { ati- } \\
\text { nine }\end{array}$ & $\underset{\mathbf{N}}{\text { Urea }}$ & $\underset{\text { lin }}{\text { Inu- }}$ & $\begin{array}{l}\text { Creat- } \\
\text { inine }\end{array}$ & $\begin{array}{l}\text { Urea } \\
\mathbf{N}\end{array}$ & Inulin & $\begin{array}{l}\text { Endog- } \\
\text { enous } \\
\text { creat- } \\
\text { inine }\end{array}$ & $\begin{array}{l}\text { Exog- } \\
\text { enous } \\
\text { creat- } \\
\text { inine }\end{array}$ & Urea & $\begin{array}{l}\text { Endog- } \\
\text { enous } \\
\text { creat- } \\
\text { inine: } \\
\text { Inulin }\end{array}$ & $\begin{array}{l}\text { Exog- } \\
\text { genous } \\
\text { creat- } \\
\text { inine: } \\
\text { Inulin }\end{array}$ & $\begin{array}{l}\text { Urea: } \\
\text { Inulin }\end{array}$ \\
\hline \multirow[t]{2}{*}{$\begin{array}{l}\text { A. C. } \% \\
21 \text { years } \\
\text { ( } V \text { factor } \$ \delta=1.03) \\
\text { February } 5,1940\end{array}$} & $\begin{array}{l}1 \\
28 \\
3 \\
4\end{array}$ & $\begin{array}{c}\text { min- } \\
\text { utes } \\
84 \\
84 \\
60 \\
60 \\
60\end{array}$ & $\begin{array}{c}c c . \\
\text { per } \\
\text { min- } \\
\text { ute } \\
2.06 \\
2.65 \\
3.57 \\
3.04\end{array}$ & $\begin{array}{c}\text { mgm. } \\
\text { per } \\
100 \\
\text { cc. } \\
\\
30.5 \\
25.5\end{array}$ & $\begin{array}{c}\text { mgm. } \\
\text { per } \\
100 \\
c c . \\
4.0\end{array}$ & $\begin{array}{c}\text { mgm. } \\
\text { per } \\
100 \\
c c . \\
33.7^{*} \\
32.7^{*} \\
34.4^{*} \\
33.6^{*}\end{array}$ & $\begin{array}{c}\text { mgm. } \\
\text { per } \\
100 \\
c c . \\
\\
125 \\
120\end{array}$ & $\begin{array}{c}\text { mgm. } \\
\text { per } \\
100 \\
c c . \\
34.2 \\
29.1 \\
22.8 \\
25.1\end{array}$ & $\begin{array}{c}\text { mgm. } \\
\text { per } \\
100 \\
c c . \\
203^{*} \\
178^{*} \\
135^{*} \\
152^{*}\end{array}$ & 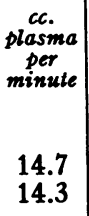 & $\begin{array}{c}c c . \\
\text { plasma } \\
\text { per } \\
\text { minute } \\
17.6 \\
19.7 \\
21.0 \\
19.2\end{array}$ & $\begin{array}{c}c c . \\
\text { plasma } \\
\text { per } \\
\text { minute }\end{array}$ & $\begin{array}{c}c c . \\
\text { plasma } \\
\text { per } \\
\text { minute } \\
12.3^{*} \\
14.4^{*} \\
14.0^{*} \\
13.7^{*}\end{array}$ & $\begin{array}{l}1.43 \\
1.34\end{array}$ & & $\begin{array}{l}0.95^{*} \\
0.96^{*}\end{array}$ \\
\hline & \multicolumn{3}{|c|}{ Average } & & & & & & & 14.5 & 19.4 & & $13.6^{*}$ & & & \\
\hline \multirow[t]{2}{*}{$\begin{array}{l}\text { Idem } \\
\text { February 10, } 1940\end{array}$} & $\begin{array}{l}1 \\
2 \\
3 \\
4 \\
5 \| \\
6 \\
7 \\
8 \ddagger t \\
9\end{array}$ & $\begin{array}{l}62 \\
60 \\
57 \\
60 \\
62 \\
64 \\
59 \\
58 \\
62\end{array}$ & $\begin{array}{l}2.28 \\
3.69 \\
3.92 \\
1.88 \\
3.30 \\
3.24 \\
2.65 \\
3.84 \\
2.52\end{array}$ & \begin{tabular}{|l|} 
\\
\\
37.7 \\
31.2 \\
28.2 \\
27.0
\end{tabular} & $\begin{array}{r} \\
8.1 \\
14.2 \\
16.2 \\
15.8 \\
15.4\end{array}$ & $\begin{array}{l}32.9^{*} \\
\\
31.8^{*} \\
31.5^{*} \\
32.5^{*} \\
32.6^{*}\end{array}$ & $\begin{array}{l}155 \\
165 \\
113 \\
128\end{array}$ & \begin{tabular}{|r}
33.0 \\
24.1 \\
21.2 \\
34.3 \\
32.3 \\
65.5 \\
108.4 \\
73.0 \\
94.9
\end{tabular} & $\begin{array}{l}188^{*} \\
114^{*} \\
111^{*} \\
203^{*} \\
131^{*} \\
127^{*} \\
152^{*} \\
110^{*} \\
155^{*}\end{array}$ & $\begin{array}{l} \\
13.5 \\
14.1 \\
15.5 \\
12.0\end{array}$ & $\begin{array}{l}18.5 \\
19.7 \\
20.1 \\
15.7\end{array}$ & $\begin{array}{l}14.9 \dagger \\
17.7 \\
17.7 \\
15.5\end{array}$ & $\begin{array}{l}13.2^{*} \\
12.9^{*} \\
13.4^{*} \\
12.0^{*} \\
13.2^{*} \\
13.0^{*} \\
12.9^{*} \\
13.1^{*} \\
12.1^{*}\end{array}$ & & $\begin{array}{l}1.10 \\
1.26 \\
1.14 \\
1.29\end{array}$ & $\begin{array}{l}0.96^{*} \\
0.91^{*} \\
0.85^{*} \\
1.01^{*}\end{array}$ \\
\hline & \multicolumn{3}{|c|}{ Average } & & & & & & & 13.8 & 18.5 & 16.5 & $12.9 *$ & & & \\
\hline \multirow[t]{2}{*}{$\begin{array}{l}\text { S. W. } \$ \\
4 \text { years } \\
(V \text { factor } \$ \delta=2.95) \\
\text { February } 13,1940\end{array}$} & $\begin{array}{l}1 \\
2 \\
3 \\
4 \ddagger \\
5 \\
6 \\
7 \ddagger \ddagger \\
8\end{array}$ & $\begin{array}{l}57 \\
55 \\
61 \\
62 \\
68 \\
54 \\
62 \\
53\end{array}$ & $\begin{array}{l}2.28 \\
4.07 \\
2.13 \\
1.90 \\
2.17 \\
2.41 \\
2.09 \\
2.50\end{array}$ & $\begin{array}{l} \\
44.1 \\
39.8 \\
35.8 \\
32.0\end{array}$ & \begin{tabular}{|r|}
2.7 \\
\\
4.3 \\
10.4 \\
13.5 \\
12.8 \\
12.0
\end{tabular} & $\begin{array}{l}43.5^{*} \\
42.6^{*} \\
46.0^{*} \\
44.4^{*}\end{array}$ & $\begin{array}{l}282 \\
205 \\
205 \\
140\end{array}$ & $\begin{array}{r}15.2 \\
9.7 \\
15.5 \\
35.0 \\
82.7 \\
88.4 \\
94.5 \\
76.8\end{array}$ & $\begin{array}{l}223^{*} \\
143^{*} \\
224^{*} \\
238^{*} \\
193^{*} \\
174^{*} \\
212^{*} \\
193^{*}\end{array}$ & \begin{tabular}{|l} 
\\
13.9 \\
12.4 \\
12.0 \\
10.9
\end{tabular} & $\begin{array}{l}12.8 \\
14.7 \\
12.3\end{array}$ & $\begin{array}{l}17.2 \dagger \\
15.7 \\
15.4 \\
16.3\end{array}$ & \begin{tabular}{|r}
$11.4^{*}$ \\
$13.0^{*}$ \\
$10.7^{*}$ \\
$10.4^{*}$ \\
$9.6^{*}$ \\
$9.9^{*}$ \\
$9.6^{*}$ \\
$10.5^{*}$
\end{tabular} & & $\begin{array}{l}1.24 \\
1.27 \\
1.28 \\
1.50\end{array}$ & $\begin{array}{l}0.69^{*} \\
0.80^{*} \\
0.80^{*} \\
0.96^{*}\end{array}$ \\
\hline & \multicolumn{3}{|c|}{ Average } & & & & & & & 12.3 & 13.3 & 16.1 & $10.6^{*}$ & & & \\
\hline
\end{tabular}


TABLE II (Continued)

\begin{tabular}{|c|c|c|c|c|c|c|c|c|c|c|c|c|c|c|c|c|}
\hline \multirow[b]{2}{*}{ Subject } & \multirow[b]{2}{*}{ Period } & \multirow[b]{2}{*}{$\begin{array}{l}\text { Dur- } \\
\text { ation }\end{array}$} & \multirow[b]{2}{*}{$\begin{array}{l}\text { Urine } \\
\text { flow } \\
V \$ 8\end{array}$} & \multicolumn{3}{|c|}{ Plasma levels } & \multicolumn{3}{|c|}{ Urine levels } & \multicolumn{4}{|c|}{ Clearances } & \multicolumn{3}{|c|}{ Clearance ratios } \\
\hline & & & & Inu- & \begin{tabular}{|c} 
Cre- \\
ati- \\
nine
\end{tabular} & $\stackrel{\text { Urea }}{\mathbf{N}}$ & $\begin{array}{l}\text { Inu- } \\
\text { lin }\end{array}$ & $\begin{array}{c}\text { Creat- } \\
\text { inine }\end{array} \mid$ & Urea & Inulin & $\begin{array}{l}\text { Endog- } \\
\text { enous } \\
\text { creat- } \\
\text { inine }\end{array}$ & \begin{tabular}{|l} 
Exog- \\
enous \\
creat- \\
inine
\end{tabular} & Urea & $\begin{array}{l}\text { Endog- } \\
\text { enous } \\
\text { creat- } \\
\text { inine: } \\
\text { Inulin }\end{array}$ & \begin{tabular}{|l|} 
Exog- \\
enous \\
creat- \\
inine: \\
Inulin
\end{tabular} & $\begin{array}{l}\text { Urea: } \\
\text { Inulin }\end{array}$ \\
\hline \multirow[t]{2}{*}{$\begin{array}{l}\text { J. C. } \sigma^{7} \\
7 \text { years } \\
(V \text { factor } \$ \delta=2.26) \\
\text { January } 29,1940\end{array}$} & $\begin{array}{l}1 \\
2 \\
3 \S \\
4 \\
5\end{array}$ & 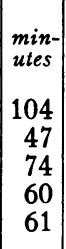 & $\begin{array}{c}c c . \\
\text { per } \\
\text { min- } \\
\text { une } \\
2.77 \\
4.23 \\
4.70 \\
4.74 \\
3.63\end{array}$ & $\begin{array}{c}\mathrm{mgm} . \\
\text { per } \\
100 \\
c c . \\
\\
\\
\\
63.3 \\
52.7\end{array}$ & $\begin{array}{c}\text { mgm. } \\
\text { per } \\
100 \\
c c . \\
2.0 \\
1.9 \\
\\
\\
\\
\end{array}$ & $\begin{array}{c}m g m . \\
\text { per } \\
100 \\
c c . \\
38.1^{*} \\
\\
37.0^{*} \\
37.2^{*} \\
36.9^{*}\end{array}$ & $\begin{array}{c}\mathrm{mgm} . \\
\text { per } \\
100 \\
c c . \\
\\
\\
260 \\
240\end{array}$ & $\begin{array}{c}\mathrm{mgm} . \\
\text { per } \\
100 \\
c c . \\
19.0 \\
12.5 \\
11.2 \\
11.1 \\
12.9\end{array}$ & $\begin{array}{c}\mathrm{mgm} . \\
\text { per } \\
100 \\
c c . \\
227^{*} \\
136^{*} \\
130^{*} \\
121^{*} \\
149^{*}\end{array}$ & $\begin{array}{c}c c . \\
\text { plasma } \\
\text { per } \\
\text { minute }\end{array}$ & \begin{tabular}{|c|}
$c c$. \\
plass \\
per \\
minute \\
25.8 \\
27.8 \\
27.1 \\
26.1 \\
23.3
\end{tabular} & $\begin{array}{c}c c . \\
\text { plasma } \\
\text { per } \\
\text { minute }\end{array}$ & \begin{tabular}{|c} 
plasma \\
per \\
minute \\
$16.5^{*}$ \\
$15.2^{*}$ \\
$16.5^{*}$ \\
$15.4^{*}$ \\
$14.5^{*}$
\end{tabular} & $\begin{array}{l}1.33 \\
1.41\end{array}$ & & $\begin{array}{l}0.79^{*} \\
0.88^{*}\end{array}$ \\
\hline & \multicolumn{9}{|c|}{ Average } & 18.0 & 25.9 & & $15.6^{*}$ & & & \\
\hline \multirow[t]{2}{*}{$\begin{array}{l}\text { Idem } \\
\text { Februarv } 1,1940\end{array}$} & $\begin{array}{l}1 \\
2 \\
3 * * \\
4 \S \\
5 \\
6 \ddagger \ddagger \\
7\end{array}$ & \begin{tabular}{|r|}
101 \\
80 \\
54 \\
61 \\
60 \\
63 \\
57
\end{tabular} & $\begin{array}{l}3.14 \\
4.74 \\
5.35 \\
4.70 \\
5.63 \\
4.03 \\
5.05\end{array}$ & $\begin{array}{l} \\
60.5 \\
50.0 \\
44.4\end{array}$ & $\begin{array}{r}2.0 \\
9.9 \\
18.1 \\
18.0 \\
17.2 \\
16.7\end{array}$ & $\begin{array}{l}39.6^{*} \\
38.8^{*} \\
37.1^{*} \\
41.0^{*} \\
41.9^{*}\end{array}$ & $\begin{array}{l}200 \\
220 \\
140\end{array}$ & \begin{tabular}{|r}
15.4 \\
8.9 \\
52.4 \\
107.2 \\
96.0 \\
116.0 \\
88.7
\end{tabular} & $\begin{array}{l}120^{*} \\
118^{*} \\
126^{*} \\
115^{*} \\
162^{*} \\
135^{*}\end{array}$ & $\begin{array}{l} \\
18.5 \\
17.7 \\
16.0\end{array}$ & $\begin{array}{l}24.2 \\
21.2\end{array}$ & $\begin{array}{l}28.2 \dagger \\
27.8 \dagger \\
30.0 \\
27.1 \\
26.9\end{array}$ & $\begin{array}{l}14.4^{*} \\
15.9^{*} \\
15.2^{*} \\
17.5^{*} \\
16.0^{*} \\
16.3^{*}\end{array}$ & & $\begin{array}{l}1.62 \\
1.53 \\
1.68\end{array}$ & $\begin{array}{l}0.95^{*} \\
0.90^{*} \\
1.02^{*}\end{array}$ \\
\hline & \multicolumn{9}{|c|}{ Average } & 17.4 & 22.7 & 28.0 & $15.9^{*}$ & & & \\
\hline \multirow[t]{2}{*}{$\begin{array}{l}\text { Idem } \\
\text { March } 7,1940\end{array}$} & $\begin{array}{l}1 \\
2 \\
3 \\
4 \pi \\
5 \S \\
6 \\
7 \\
8+\dagger\end{array}$ & \begin{tabular}{|r}
61 \\
60 \\
76 \\
54 \\
66 \\
59 \\
70 \\
140
\end{tabular} & \begin{tabular}{|l|}
9.05 \\
6.68 \\
0.38 \\
6.84 \\
3.62 \\
3.58 \\
3.60 \\
2.99
\end{tabular} & $\begin{array}{l} \\
\\
82.0 \\
74.0 \\
60.0\end{array}$ & $\begin{array}{l}4.6 \\
10.0 \\
17.1 \\
17.6 \\
16.6 \\
16.0\end{array}$ & $\begin{array}{l}98.6 \\
92.5 \\
96.4\end{array}$ & $\begin{array}{l}205 \\
170 \\
195\end{array}$ & $\begin{array}{l}18.1 \\
17.5 \\
12.1 \\
21.5 \\
51.3 \\
50.6 \\
46.9 \\
55.9\end{array}$ & $\begin{array}{l}260 \\
247 \\
152 \\
203 \\
182 \\
176 \\
178 \\
222\end{array}$ & $\begin{array}{l}9.0 \\
8.3 \\
9.7\end{array}$ & $\begin{array}{l}35.9 \\
25.5\end{array}$ & $\begin{array}{l}10.9 \dagger \\
10.4 \\
10.2 \\
10.5\end{array}$ & \begin{tabular}{|r|}
23.5 \\
16.5 \\
1.3 \\
13.9 \\
6.5 \\
6.4 \\
6.9 \\
6.9
\end{tabular} & & $\begin{array}{l}1.16 \\
1.23 \\
1.08\end{array}$ & $\begin{array}{l}0.71 \\
0.83 \\
0.71\end{array}$ \\
\hline & \multicolumn{9}{|c|}{ Average } & - & & - & - & & & \\
\hline
\end{tabular}

* See asterisked footnote Table I.

$\dagger$ Plasma creatinine level rising during this period.

I Inulin 3.5 grams given intravenously and creatinine 1 gram given by mouth during this period.

Inulin 5 grams given intravenously during this period.

II Inulin 5 grams given intravenously and creatinine 4 grams given by mouth during this period.

I Creatinine 2 grams given by mouth during this period.

** Creatinine 5 grams given by mouth during this period.

t† Casein hydrolysate 5 grams given intravenously during this period.

t† Casein hydrolysate 10 grams given intravenously during this period.

\$\& See footnote $\ddagger \ddagger$, Table $\mathrm{I}$.

\section{CALCULATIONS}

The urea, creatinine, and inulin clearances were calculated as the number of cc. of plasma cleared per minute per 1.73 square meters of surface area. The formula ${ }^{3}$

${ }^{3}$ The general clearance formula, introduced by M $\phi$ ller, McIntosh, and Van Slyke (14), is:

$$
\text { Clearance }=\frac{U V}{P} \text {. }
$$

$U$ and $P$ are, respectively, the concentrations in urine and plasma of the excreted substance-urea, creatinine, or inulin, etc.-and $V$ is the urine flow expressed as cc. per minute per 1.73 square meters of body surface. The of Møller, McIntosh and Van Slyke (14) was used. We have termed "endogenous" the creatinine clearances which were measured without administration of creatinine,

use of surface area in this calculation, as in the calculation of McIntosh, M $\phi 1 l e r$ and Van Slyke (15), makes the clearance formula give the same normal values for infants and children as for adults $(15,16)$. The surface area used in the calculation is estimated from the height and age of the child, as described by McIntosh et al. (15).

When the urine volume is above $2 \mathrm{cc}$. per minute per 1.73 square meters of body area, the urea clearance in man is independent of volume change; hence, urea clearances with $V$ above $2 \mathrm{cc}$. have been termed "maximum" 
TABLE III

Control subjects (recovered group). Comparison of inulin, creatinine, and urea clearances

\begin{tabular}{|c|c|c|c|c|c|c|c|c|c|c|c|c|c|c|c|}
\hline \multirow[b]{2}{*}{ Subject } & \multirow[b]{2}{*}{ Period } & \multirow[b]{2}{*}{$\underset{\substack{\text { Dura- } \\
\text { tion }}}{ }$} & \multirow[b]{2}{*}{$\begin{array}{c}\text { Urine } \\
\text { flow } \\
V \S\end{array}$} & \multicolumn{3}{|c|}{ Plasma levels } & \multicolumn{3}{|c|}{ Urine levels } & \multicolumn{4}{|c|}{ Clearances } & \multicolumn{2}{|c|}{$\begin{array}{c}\text { Clearance } \\
\text { ratios }\end{array}$} \\
\hline & & & & Inulin & $\begin{array}{l}- \text { Creat- } \\
\text { inine }\end{array}$ & $\underset{\mathbf{N}}{\text { Urea }}$ & Inulin & $\begin{array}{l}\text { Creat- } \\
\text { inine }\end{array}$ & $\underset{\mathbf{N}}{\text { Urea }}$ & Inulin & $\begin{array}{c}\text { Endog- } \\
\text { enous } \\
\text { creat- } \\
\text { inine }\end{array}$ & $\begin{array}{l}\text { Exog- } \\
\text { enous } \\
\text { creat- } \\
\text { inine }\end{array}$ & Urea & $\begin{array}{l}\text { Erog- } \\
\text { enous } \\
\text { creat- } \\
\text { inine: } \\
\text { Inulin }\end{array}$ & $\begin{array}{l}\text { Urea: } \\
\text { Inulin }\end{array}$ \\
\hline \multirow[t]{2}{*}{$\begin{array}{l}\text { H. G. }{ }^{+} \\
5 \text { years } \\
(V \text { factor } \delta=2.10) \\
\text { April } 22,1940\end{array}$} & $\begin{array}{c}1 \\
2 \\
3 \\
4 \ddagger \\
5^{*} \\
6 \\
7 \\
8 \\
9 \\
10\end{array}$ & 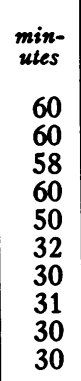 & $\begin{array}{c}c c . \\
\text { per } \\
\text { minute } \\
1.47 \\
9.11 \\
2.97 \\
3.25 \\
5.46 \\
1.71 \\
3.42 \\
13.50 \\
15.00 \\
5.04\end{array}$ & $\begin{array}{c}\text { mgm. } \\
\text { per } \\
100 \\
c c .\end{array}$ & $\begin{array}{c}\text { mgm. } \\
\text { per } \\
100 \\
c c . \\
0.48 \\
\\
\\
0.70 \\
5.98 \\
13.25 \\
11.12 \\
8.92 \\
7.20 \\
6.14\end{array}$ & $\begin{array}{c}\text { mgm. } \\
\text { per } \\
100 \\
c c . \\
14.6 \\
\end{array}$ & $\begin{array}{c}\text { mgm. } \\
\text { per } \\
100 \\
c c . \\
\\
\\
\\
\\
4160 \\
1200 \\
157 \\
84 \\
165\end{array}$ & \begin{tabular}{|c|} 
mgm. \\
per \\
100 \\
$c c$. \\
49.6 \\
6.0 \\
9.4 \\
18.1 \\
162.0 \\
1300.0 \\
570.0 \\
122.0 \\
88.8 \\
165.0
\end{tabular} & $\begin{array}{r}\text { mgm. } \\
\text { per } \\
100 \\
c c . \\
1170 \\
121 \\
150 \\
322 \\
152 \\
740 \\
278 \\
78 \\
66 \\
144\end{array}$ & $\begin{array}{r}146 \\
147 \\
113 \\
93 \\
87\end{array}$ & $\begin{array}{c}c c . \\
\text { plasma } \\
\text { per } \\
\text { minute } \\
151 \\
112 \\
58\end{array}$ & $\begin{array}{l}167 \\
176 \\
185 \\
185 \\
135\end{array}$ & $\begin{array}{c}\text { cc. } \\
\text { plasma } \\
\text { per } \\
\text { minute } \\
138 \\
75 \\
30 \\
72 \\
62 \\
103 \\
76 \\
78 \\
79 \\
68\end{array}$ & $\begin{array}{l}1.14 \\
1.20 \\
1.64 \\
1.99 \\
1.55\end{array}$ & $\begin{array}{l}0.71 \\
0.52 \\
0.69 \\
0.85 \\
0.78\end{array}$ \\
\hline & \multicolumn{3}{|c|}{ Average } & & & & & & & 117 & 107 & 170 & 78 & & \\
\hline \multirow[t]{2}{*}{$\begin{array}{l}\text { B. D. } 9 \\
6 \text { years } \\
\text { ( } V \text { factor } \$=2.12) \\
\text { June } 21,1940\end{array}$} & $\begin{array}{l}1 \\
2 \\
3 \dagger \\
4^{*} \\
5 \\
6 \\
7 \\
8\end{array}$ & $\begin{array}{r}60 \\
51 \\
123 \\
63 \\
30 \\
30 \\
31 \\
29\end{array}$ & $\begin{array}{l}0.92 \\
4.63 \\
0.67 \\
7.42 \\
2.26 \\
2.48 \\
4.45 \\
4.09\end{array}$ & $\begin{array}{l}57.0 \\
28.0 \\
17.5 \\
12.5\end{array}$ & \begin{tabular}{|r|}
8.21 \\
10.16 \\
10.34 \\
9.40 \\
8.38
\end{tabular} & $\begin{array}{r}11.8 \\
11.5 \\
11.2 \\
10.9 \\
9.9\end{array}$ & $\begin{array}{r}3870 \\
1725 \\
590 \\
450\end{array}$ & $\begin{array}{r}58.2 \\
11.7 \\
\\
197.0 \\
626.0 \\
737.0 \\
382.0 \\
387.0\end{array}$ & $\begin{array}{l}658 \\
328 \\
451 \\
148 \\
339 \\
324 \\
171 \\
186\end{array}$ & $\begin{array}{l}154 \\
152 \\
150 \\
147\end{array}$ & $\begin{array}{l}116 \\
117\end{array}$ & $\begin{array}{l}176 \\
180 \\
189\end{array}$ & $\begin{array}{r}69 \\
117 \\
40 \\
93 \\
67 \\
72 \\
70 \\
77\end{array}$ & $\begin{array}{l}1.16 \\
1.20 \\
1.29\end{array}$ & $\begin{array}{l}0.43 \\
0.47 \\
0.47 \\
0.52\end{array}$ \\
\hline & \multicolumn{3}{|c|}{ Average } & \multicolumn{3}{|c|}{ - } & & & & 151 & 116 & 182 & 76 & & \\
\hline
\end{tabular}

* Inulin 10 grams given intravenously during this period. $\uparrow$ Creatinine 4 grams given by mouth during this period.

and "exogenous" those which were determined after the blood creatinine content was increased by the feeding of creatinine. The values for the plasma concentrations of inulin and creatinine, in experiments where these substances were administered, were estimated for the midpoint of each period by interpolation on a graph, plasma concentration being plotted arithmetically against time. In each instance, the reported values for the inulin clearances were estimated from data obtained while the plasma level of inulin was falling; calculations of exogenous creatinine clearances were likewise made from data ob-

clearances (14). When $V$ is between 0.5 and 2 cc., the urea clearance has been found to vary as the square root of the urine volume (14). Hence, for volumes within this range, the maximum urea clearance is calculated as $\frac{U}{P} \times V \times \sqrt{\frac{2}{V}}$ or $\frac{U}{P} \sqrt{2 V}$ in this paper. The clearances of inulin and creatinine are calculated simply as $\frac{U V}{P}$ for all urine volumes, since it was noted that a fall of $V$ to the range between 2 and $0.5 \mathrm{cc}$. did not decrease the clearance of inulin or creatinine. $\neq$ Creatinine 5 grams given by mouth during this period. $\$$ See footnote $¥ \ddagger$, Table I.

tained while the plasma creatinine concentration was falling, except where it is specifically stated otherwise in the tables. Since blood urea changed but little during the experiment, the plasma urea value directly obtained at the end of each period was used in calculating the urea clearance for that period.

\section{RESULTS}

\section{Comparison of urea, inulin and creatinine clearances}

The results are given in detail, with respect to each patient of the 3 groups, in Tables I, II and III. In general, all clearances were affected similarly in each group of patients. Those patients with a high urea clearance had elevated creatinine and inulin clearances. In the control group all three clearances were within the usual ranges of normal values $(4,15,16,17)$. In the lowclearance group all three clearances were depressed. The consistency of the results in each 
group is best demonstrated by the ratio of urea clearance to inulin clearance, which is also shown in Tables I, II, and III. The use of the ratio for comparative purposes compensates in part for divergencies in calculated results due to incomplete voiding of urine.

J. C. (Table II) failed to excrete ingested water during the experiment of March 7. An abrupt drop in clearances occurred during the experiment. Soon after the close of Period 8 the patient manifested a generalized convulsion which we believe was a result of water retention.

\section{Administration of casein hydrolysate}

The intravenous administration of casein hydrolysate to low-clearance patients was not followed by a rise in the clearance values. When it was given to S. G. he developed a severe chill and hyperpyrexia, with the diverse effects on the clearances noted in Table I. He had tolerated similar injections without reactions during the preceding 6 months.

\section{DISCUSSION}

\section{High clearance and glomerular filtration}

Our patients with high urea clearances showed similarly high inulin clearances. Therefore, if we assume with Chasis and Smith (18) that the inulin clearance is an accurate index of glomerular filtration, we may conclude that in our patients the rate of formation of glomerular filtrate was abnormally rapid.

The question remains, whether the doubling of the rate of glomerular filtration was due (1) to doubling of the renal blood flow, with a normal filtration fraction of about 20 per cent $(4,19)$; (2) to a doubling of the filtered fraction of the plasma water, with a concomitant rise in the " extraction percentage" (19) of inulin and urea; or (3) to a combination of both mechanisms. One might expect that the hypoproteinemia of nephrosis, with resultant decrease in plasma oncotic pressure, would induce glomerular filtration of an increased fraction of the plasma water. We have, however, been unable to find any consistent correlation between low plasma albumin and high urea clearance, since we have observed the high clearance to persist long after normal plasma protein concentration has been regained. It appears, therefore, that some cause more dominant than hypoproteinemia is chiefly responsible."

\section{Tubular excretion of creatinine}

We have estimated the relative output of creatinine by glomerular filtration and tubular excretion on the assumption that the inulin clearance measured glomerular filtration. From the data of each experiment the rate of total creatinine excretion and the rate of filtration of creatinine (inulin clearance $X$ plasma creatinine concentration) were calculated as mgm. per minute per 1.73 square meters of body surface and plotted for each period against the plasma creatinine concentration for that period. The periods both with and without creatinine feeding were included. With uniformity the curves obtained approximated straight lines, both for observed total creatinine excretion and for estimated glomerular filtration, at plasma levels up to $10 \mathrm{mgm}$. per 100 cc.

For numerical comparisons of the different subjects, excretions have been calculated for a constant plasma creatinine concentration of $10 \mathrm{mgm}$. per $100 \mathrm{cc}$., or $0.1 \mathrm{mgm}$. per cc. The calculations have been made as follows:

(a) Total mgm. creatinine excreted per minute $=($ cc. plasma cleared of creatinine per minute) $\times 0.1$

(b) Mgm. creatinine filtered by glomeruli per minute $=(\mathrm{cc}$. plasma cleared of inulin per minute) $\times 0.1$

(c) Mgm. creatinine excreted by tubules per minute $=a-b$.

The values for plasma creatinine clearance used in formula $a$ and inulin clearance used in formula $b$ are the means of the determinations in each subject.

4 There is one type of control that our data lack, viz., the estimation of urea clearances on entirely normal children placed on the same régime of diet (salt poor, 3 grams of protein per kilo) and activity as our patients. The possibility that under these conditions normal children might show higher than ordinary clearances is not excluded by our data, nor by any that we have found in the literature. The absence of such high values in normal children on unrestricted diets (16) makes their occurrence seem improbable, but one cannot say that it is absolutely excluded. 
The results of the calculations are given in $\mathrm{Ta}-$ ble IV. They indicate that the estimated tubular excretion of creatinine in the 3 groups parallels glomerular filtration, the mean tubular excretion of the high-clearance group being $12.9 \mathrm{mgm}$. per minute and that of the low-clearance group 0.6 mgm., compared with $4.2 \mathrm{mgm}$. for the controls.

\section{TABLE IV}

Rate of excretion of creatinine by all subjects estimated for a plasma creatinine concentration of $10 \mathrm{mgm}$. per $100 \mathrm{cc}$., corrected to a body surface area of 1.73 square meters

\begin{tabular}{|c|c|c|c|}
\hline Patient & Total & Filtered & Secreted \\
\hline $\begin{array}{l}\text { Recovered group } \\
\text { H. G. } \\
\text { B. D. }\end{array}$ & $\begin{array}{l}\underset{\text { mgm. per }}{\text { minute }} \\
17.0 \\
18.2\end{array}$ & 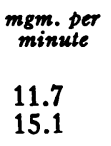 & $\begin{array}{c}\underset{\text { mgm. per }}{\text { minute }} \\
\\
5.3 \\
3.1\end{array}$ \\
\hline Average & 17.6 & 13.4 & 4.2 \\
\hline $\begin{array}{l}\text { High-clearance group } \\
\text { R. Q. March 11, } 1940 \\
\text { S. G. } \\
\text { R. M. }\end{array}$ & $\begin{array}{l}29.7 \\
36.6 \\
32.0\end{array}$ & $\begin{array}{l}19.3 \\
17.1 \\
23.3\end{array}$ & $\begin{array}{r}10.4 \\
19.5 \\
8.7\end{array}$ \\
\hline Average & 32.8 & 19.9 & 12.9 \\
\hline $\begin{array}{l}\text { Low-clearance group } \\
\text { J. C. February 1, } 1940 \\
\text { S. W. } \\
\text { A. C. }\end{array}$ & $\begin{array}{l}2.80 \\
1.05^{*} \\
1.61 \\
1.65\end{array}$ & $\begin{array}{l}1.74 \\
0.90^{*} \\
1.23 \\
1.38\end{array}$ & $\begin{array}{l}1.06 \\
0.15^{*} \\
0.38 \\
0.27\end{array}$ \\
\hline Average & 2.02 & 1.45 & 0.57 \\
\hline
\end{tabular}

* Not included in average - see text.

\section{CONCLUSIONS}

The persistent, abnormally high urea clearance, as great as 150 to 200 per cent of normal average, observed in certain children with the nephrotic syndrome, is a manifestation of generally increased renal excretory activity, since the inulin and creatinine clearances are also elevated above normal to approximately the same degree.

Insofar as tubular activity can be estimated from the ratio of exogenous creatinine clearance to inulin clearance, it appears that this activity is as much accelerated as is glomerular filtration.

Intravenous injection of amino acids did not increase the clearances in patients with diminished renal function.

\section{BIBLIOGRAPHY}

1. Farr, L. E., The effect of dietary protein on the urea clearance of children with nephrosis. J. Clin. Invest., 1936, 15, 703.
2. Goldring, W., and others, The influence of protein intake on the urea clearance in normal man. J. Clin. Invest., 1934, 13, 743.

3. Cope, C. L., Studies of urea excretion. VIII. The effects on the urea clearance of changes in protein and salt contents of the diet. J. Clin. Invest., 1933, $12,567$.

4. Smith, H. W., Physiology of the renal circulation. The Harvey Lectures, 1940, Ser. 35, 166.

5. Farr, L. E., Minimal nitrogen requirements of children with the nephrotic syndrome. Effect of the administration of a growth-promoting anterior pituitary extract. Am. J. Dis. Child., 1940, 60, 1324.

6. Farr, L. E., Emerson, K., Jr., and Futcher, P. H., The comparative nutritive efficiency of intravenous amino acids and dietary protein in children with the nephrotic syndrome. J. Pediat., 1940, 17, 595.

7. Farr, L. E., The intravenous administration of small doses of casein hydrolysate to nephrotic children and its effect upon the nitrogen balance and plasma amino acid level. J. Pediat., 1940, 16, 679.

8a. Van Slyke, D. D., The manometric determination of urea in blood and urine by the hypobromite reaction. J. Biol. Chem., 1929, 83, 449.

b. Van Slyke, D. D., and Kugel, V. H., Improvements in manometric micro-Kjeldahl and blood urea methods. J. Biol. Chem., 1933, 102, 489.

9. Van Slyke, D. D., Determination of urea by gasometric measurement of the carbon dioxide formed by the action of urease. J. Biol. Chem., 1927, 73, 695.

10. Summerson, W. H., A simplified test-tube photoelectric colorimeter, and the use of the photoelectric colorimeter in colorimetric analysis. J. Biol. Chem., 1939, 130, 149.

11. Miller, B. F., and Winkler, A. W., The renal excretion of endogenous creatinine in man. Comparison with exogenous creatinine and inulin. J. Clin. Invest., 1938, 17, 31.

12. Miller, B. F., and Dubos, R., Determination by a specific, enzymatic method of the creatinine content of blood and urine from normal and nephritic individuals. J. Biol. Chem., 1937, 121, 457.

13. Alving, A. S., Rubin, J., and Miller, B. F., A direct colorimetric method for the determination of inulin in blood and urine. J. Biol. Chem., 1939, 127, 609.

14. Mфller, E., McIntosh, J. F., and Van Slyke, D. D., Studies of urea excretion. II. Relationship between urine volume and the rate of urea excretion by normal adults. J. Clin. Invest., 1928, 6, 427.

15. McIntosh, J. F., Møller, E., and Van Slyke, D. D., Studies of urea excretion. III. The influence of body size on urea output. J. Clin. Invest., 1928, $6,467$.

16. Cullen, G. E., Nelson, W. E., and Holmes, F. E., Studies of kidney function in children. I. Urea clearance values. J. Clin. Invest., 1935, 14, 563. 
17. Hayman, J. M., Jr., Halsted, J. A., and Seyler, L. E., A comparison of the creatinine and urea clearance tests of kidney function. J. Clin. Invest., 1933, $12,861$.

18. Chasis, H., and Smith, H. W., The excretion of urea in normal man and in subjects with glomerulonephritis. J. Clin. Invest., 1938, 17, 347.

19a. Van Slyke, D. D., Hiller, A., and Miller, B. F.,
The clearance, extraction percentage and estimated filtration of sodium ferrocyanide in the mammalian kidney. Comparison with inulin, creatinine and urea. Am. J. Physiol., 1935, 113, 611.

b. Idem, The distribution of ferrocyanide, inulin, creatinine, and urea in the blood and its effect on the significance of their extraction percentages. Am. J. Physiol., 1935, 113, 629. 